\title{
Fermi Gamma-ray Space Telescope Observations of Gamma-ray Pulsars
}

\author{
P. M. Saz Parkinson for the Fermi-LAT Collaboration ${ }^{1}$ \\ Santa Cruz Institute for Particle Physics, University of California, Santa Cruz, CA 95064 \\ e-mail:pablo@scipp.ucsc.edu
}

\begin{abstract}
The Large Area Telescope on the recently launched Fermi Gamma-ray Space Telescope (formerly GLAST), with its large field of view and effective area, combined with its excellent timing capabilities, is poised to revolutionize the field of gamma-ray astrophysics. The large improvement in sensitivity over EGRET is expected to result in the discovery of many new gamma-ray pulsars, which in turn should lead to fundamental advances in our understanding of pulsar physics and the role of neutron stars in the Galaxy. Almost immediately after launch, Fermi clearly detected all previously known gamma-ray pulsars and is producing high precision results on these. An extensive radio and X-ray timing campaign of known (primarily radio) pulsars is being carried out in order to facilitate the discovery of new gamma-ray pulsars. In addition, a highly efficient time-differencing technique is being used to conduct blind searches for radio-quiet pulsars, which has already resulted in new discoveries. I present some recent results from searches for pulsars carried out on Fermi data, both blind searches, and using contemporaneous timing of known radio pulsars.
\end{abstract}

Keywords: pulsars; Fermi; blind search; PSR B1706-44; PSR J2021+3651; PSR J0010+7309; CTA 1; astronomical observations gamma-ray

PACS: $95.55 . \mathrm{Ka}$; 95.75.Wx; 95.85.Pw; 97.60.Gb

\section{INTRODUCTION}

Among the numerous significant results of the EGRET mission, on the Compton Gamma Ray Observatory (CGRO), was the firm detection of 6 gamma-ray pulsars (and marginal detection of at least three additional ones) at energies above $100 \mathrm{MeV}$. In addition to these, the COMPTEL detector, also on CGRO, detected pulsations from PSR B150958 up to $10 \mathrm{MeV}$. More than half the $\sim 300$ gamma-ray sources detected by EGRET remained unidentified at the end of the mission, and many of these (especially those lying in the Galactic Plane) are thought to be pulsars. The light curves, in various energy bands, of these seven CGRO gamma-ray pulsars are shown in Figure 1. For a detailed summary of the EGRET results, see [1]. Of these seven gamma-ray pulsars, six display clear radio pulsations, while the seventh, Geminga, is what is known as a "radio-quiet" (or at least "radio-faint") pulsar. Indeed, the entire class of radio-quiet pulsars (which until the Fermi era contained this one object) is sometimes referred to as "Gemingatype" pulsars. Differences between the radio and gamma-ray beam geometries (e.g. [2]) are thought to explain why certain pulsars are radio-loud or radio-quiet. Outer magnetosphere models predict a fan-like gamma-ray beam. The much narrower radio

${ }^{1}$ http://www-glast. stanford.edu/cgi-bin/people 


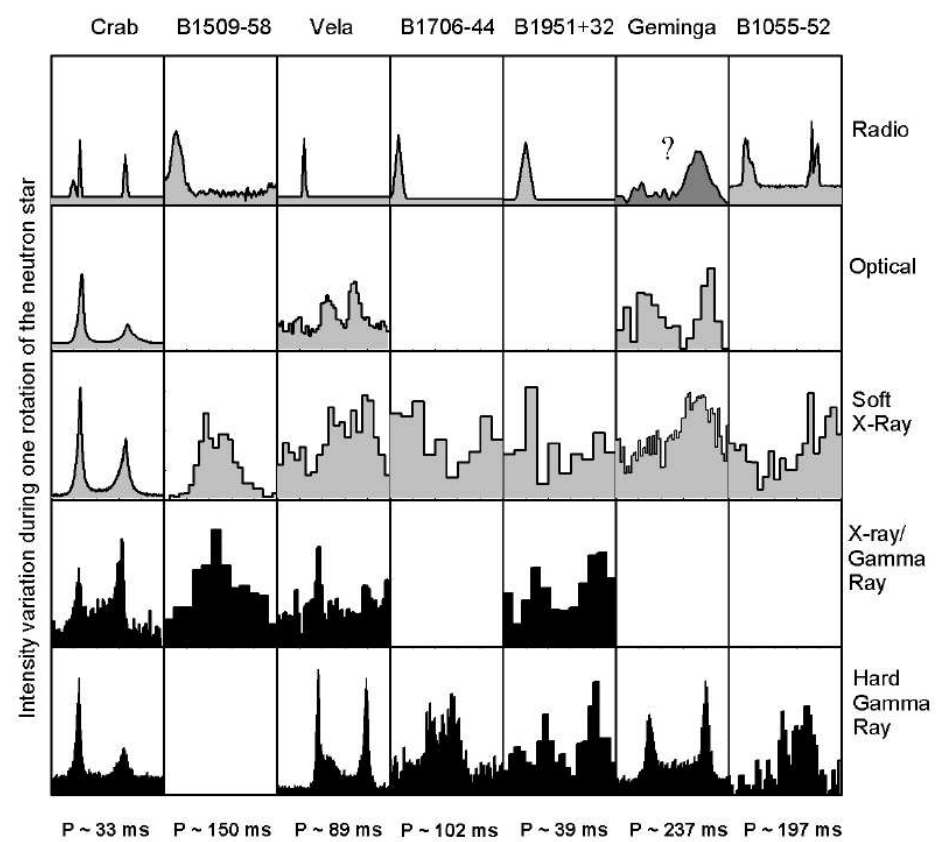

FIGURE 1. Phase-aligned folded light curves of the CGRO pulsars, at various wavelengths (from [1]).

beam, on the other hand, can often miss the Earth.

Population synthesis models of radio and gamma-ray pulsars (e.g. [3]) have been used to try to predict the number of radio-loud and radio-quiet pulsars that will be detected by the new generation of gamma-ray telescopes (AGILE and Fermi). The experimental determination of this "Geminga fraction" and a comparison with model predictions will help not only in discriminating between different models of gammaray emission, but will hopefully also serve to pin down some of the physical (and/or geometrical) parameters of the given models. Figure 2 shows, among other things, the large number of radio-loud and radio-quiet pulsars that are expected to be detected by Fermi, according to one particular population synthesis study [3].

\section{THE FERMI LARGE AREA TELESCOPE (LAT)}

The GLAST (now Fermi) satellite, consisting of the Gamma-ray Burst Monitor (GBM) and the Large Area Telescope (LAT), was launched on 11 June 2008 (see Figure 3) into a low Earth circular orbit at an altitude of $550 \mathrm{~km}$ and an inclination of $28.5^{\circ}$. The LAT [4] is a pair-production telescope with large effective area $\left(\sim 8000 \mathrm{~cm}^{2}\right)$ and field of view $(2.4 \mathrm{sr})$, sensitive to gamma rays between $20 \mathrm{MeV}$ and $>300 \mathrm{GeV}$. Although its commisioning phase (30 June to 30 July 2008) was primarily intended for instrument checkout and calibration, several scientific results have been obtained with these early data. The LAT began normal science operations on 11 August 2008, and since then has been observing mostly in survey mode, scanning the entire gamma-ray sky every three hours. The overall sensitivity of the LAT is $\sim 25$ times that of EGRET, while the angular 

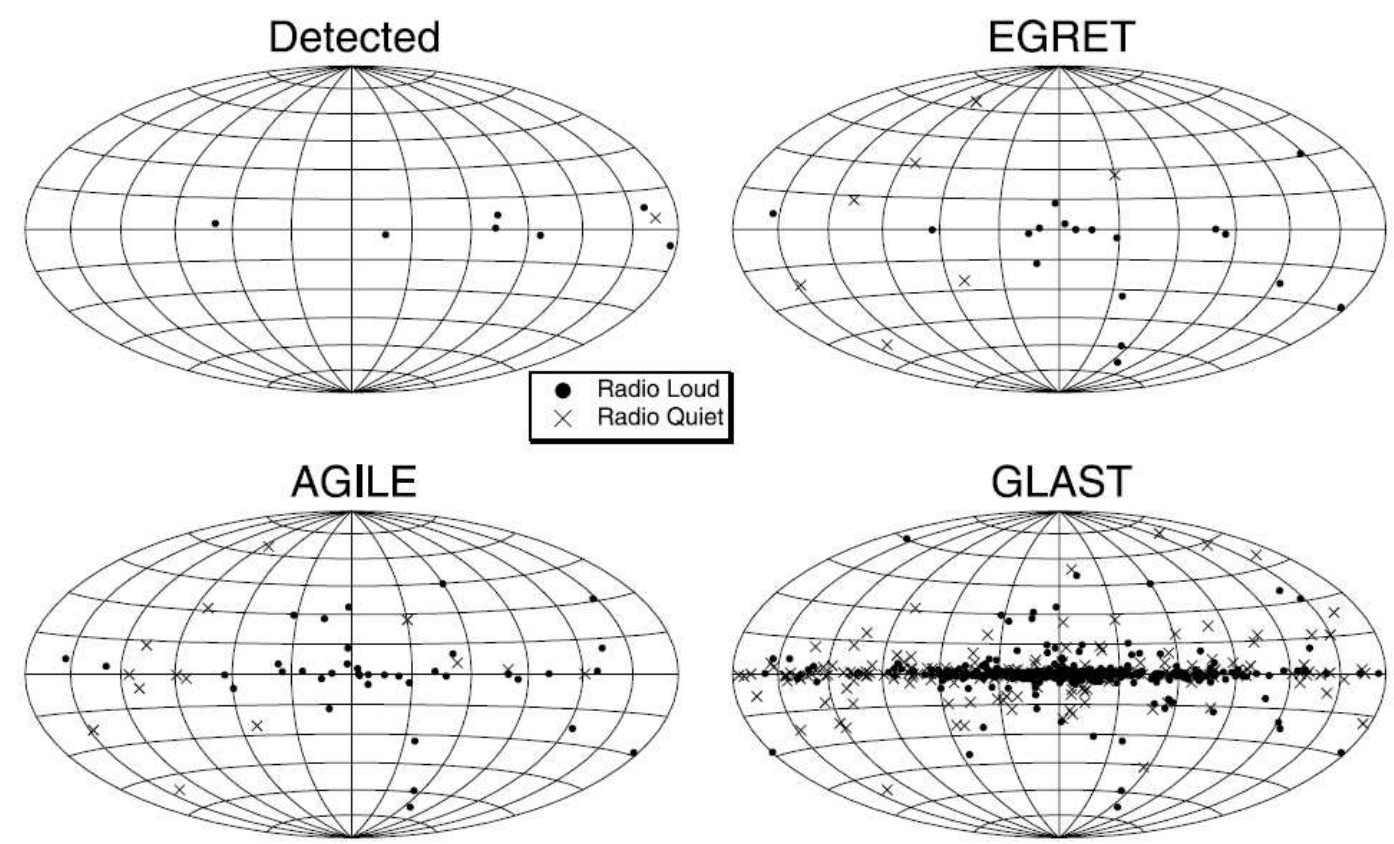

FIGURE 2. Plots showing the radio-loud (dots) and radio-quiet (crosses) pulsars detected by EGRET (top left), along with the simulated EGRET detections (top right), AGILE detections and GLAST (Fermi) detections, for a particular population synthesis study (from [3]).

resolution is also significantly improved (it ranges from $\sim 3-6^{\circ}$ at $100 \mathrm{MeV}$ to $\sim 0.1-$ $0.2^{\circ}$ at $10 \mathrm{GeV}$ ). The mission was designed with a five-year lifetime and a goal of at least ten years of operations. The scientific goals of the mission include understanding particle acceleration in Active Galactic Nuclei (AGN), pulsars, and supernova remnants (SNRs), exploring the high energy emission of Gamma-ray Bursts (GRB), and probing the nature of dark matter (see contribution by V. Vitale in these proceedings).

\section{FERMI-LAT SEARCHES FOR GAMMA-RAY PULSARS}

\section{Early observations of the EGRET pulsars}

Within the 60-day launch and early operations (L\&EO) period, the six EGRET pulsars were detected. Figure 3 shows an early LAT light curve of Geminga. The scale is not zero-suppressed, illustrating the low level of background achieved by the LAT.

Early observations of the Vela pulsar, the brightest persistent GeV source in the sky, were used to verify the excellent timing and angular resolution of the LAT and to highlight its potential for discovery in the field of pulsar astrophysics (see the contribution by M. Razzano in these proceedings, as well as the recently accepted journal article [5]). 


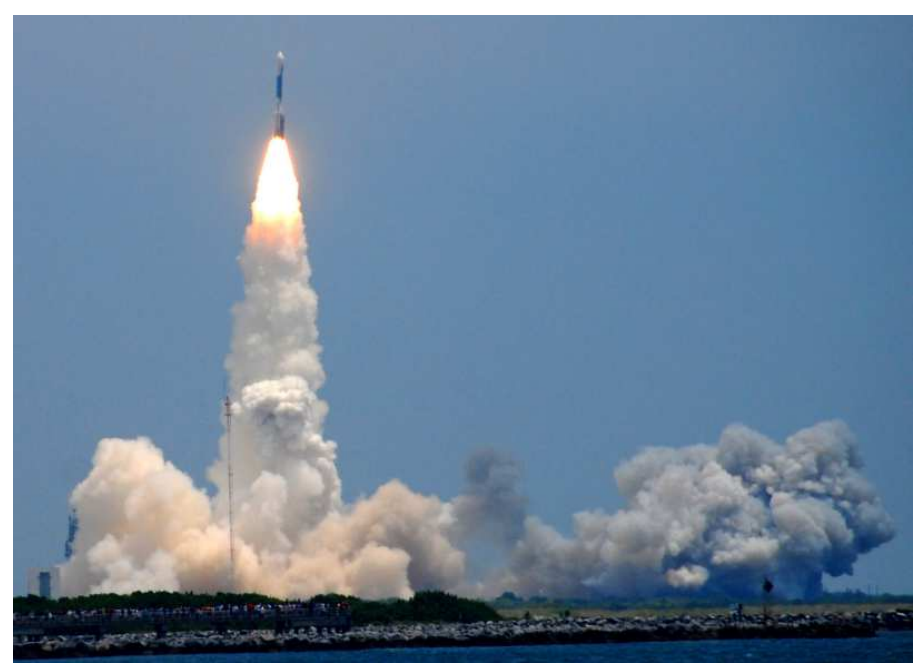

FIGURE 3. GLAST launch from Cape Canaveral aboard a Delta II rocket. (Credit: M. Ziegler)

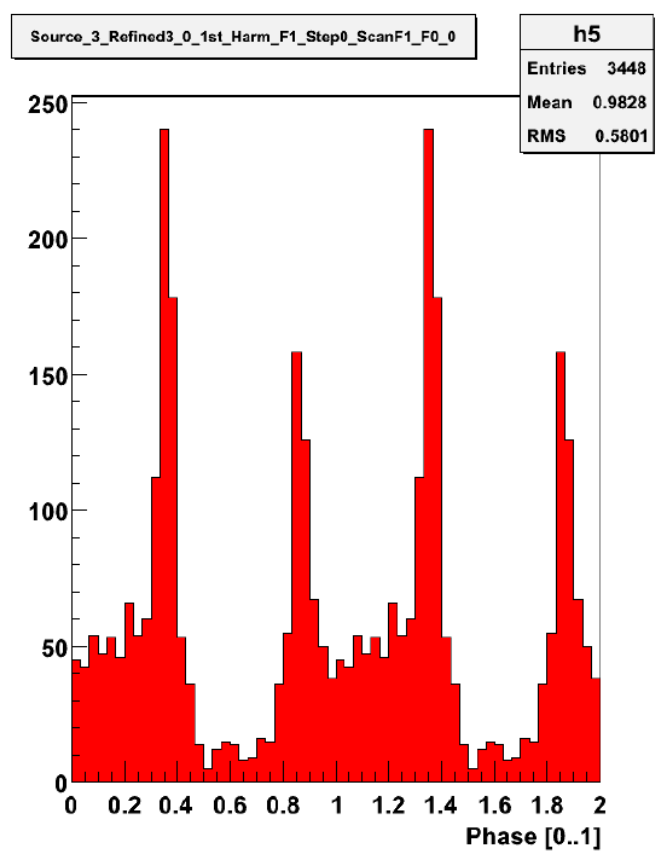

FIGURE 4. Early (commissioning phase) Fermi-LAT light curve of Geminga.

\section{Detection of a glitch in PSR B1706-44}

One of the early surprises in our study of EGRET pulsars came in the form of an apparent glitch in PSR B1706-44. Glitches are discrete changes in the rotation speed of a pulsar. They are thought to occur when a sudden transfer of angular momentum takes place from the faster rotating superfluid interior to the solid crust of the neutron star [6]. They are fairly rare, only having been detected in fewer than 50 pulsars, and they tend 


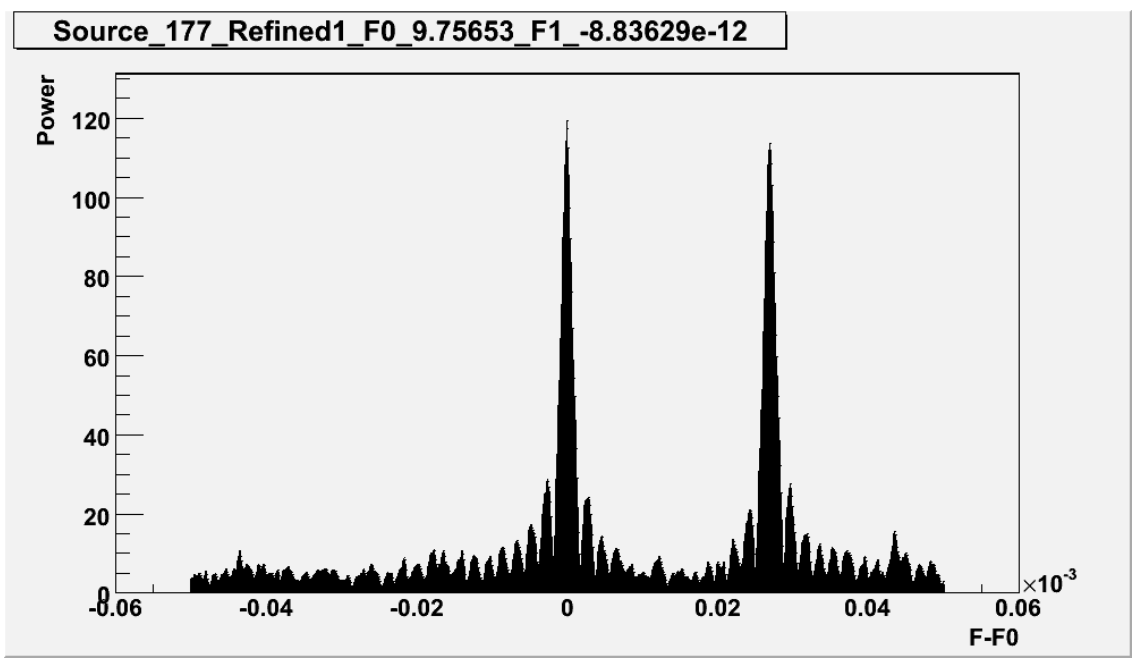

FIGURE 5. Fourier power spectrum of PSR B1706-44 ( $~ 10$ weeks of data) centered around the known frequency of the pulsar, showing peaks at both the pre-glitch and post glitch rotation frequencies.

to occur more frequently in young pulsars. The typical fractional increase in rotational frequency is $10^{-10}<\Delta f / f<5 \times 10^{-6}$ [7]. A Fourier transform of the data on PSR B1706-44 revealed two highly significant peaks. Figure 4 shows the power spectrum centered around the previously known frequency of PSR B1706-44 $(\sim 9.756532 \mathrm{~Hz})$. The second peak is at a frequency $2.8 \times 10^{-5} \mathrm{~Hz}$ higher, yielding quite a large value of $\Delta f / f \sim 2.9 \times 10^{-6}$. The data were split in two, to ascertain when the glitch took place and determine the best ephemeris for each time period (pre- and post-glitch). The different phase plots are shown in Figure 5. Due to the low flux of the source and the mode of observation of the LAT, the time of the glitch could only be narrowed down to a 22-hour window: between 08:40 on the 14th of August (MJD 54692.36) and 06:00 on the 15th of August (MJD 54693.25). While this pulsar has been known to glitch in the past (e.g. 1995 [8]), this represents the first time that a glitch has been detected in gamma rays. Follow-up radio observations are planned, to confirm the glitch.

\section{Search for gamma-ray pulsations from known pulsars}

Gamma-ray sources are inherently dim and result in very sparse data sets. The detection of gamma-ray pulsations therefore requires observations spanning long periods of time (up to years). During such long time spans, many pulsars will experience irregularities in their timing behavior, such as "timing noise" or glitches. This makes it necessary to observe pulsars regularly to obtain timing solutions valid for the entire period of observation. In anticipation of this, a comprehensive pulsar monitoring campaign (also known as the "pulsar timing consortium") was organized prior to the launch of Fermi to obtain contemporaneous timing solutions for a large (and increasing) number (currently several hundred) of the brightest (as measured by spin-down power) known radio and X-ray pulsars [9]. The first results from these efforts came in the form of the detection, 

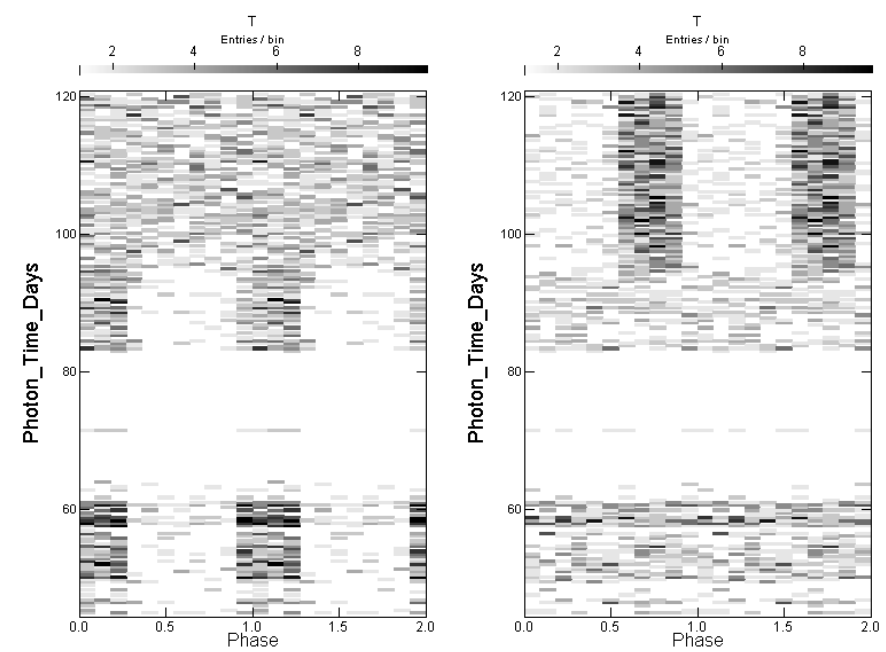

FIGURE 6. PSR B1706-44 phase plots Left - Using the "pre-glitch" timing solution. Right - Using the "post-glitch" timing solution.

during the first weeks of the commissioning phase, of pulsations from PSR J2021+3651 (also known as the "Dragonfly", a relatively recently discovered radio pulsar coincident with an unidentified EGRET source [10]). The discovery of gamma-ray pulsations from this source was recently reported using AGILE observations carried out between 2 November 2007 and 30 June 2008 [11]. A detailed publication containing the Fermi results is in preparation for submission to the Astrophysical Journal.

\section{Blind searches for gamma-ray pulsars}

In addition to searching for gamma-ray pulsations from known radio (or X-ray) pulsars, one of the goals of Fermi is to discover new pulsars altogether. A "pulsar search consortium" (similar in nature to the "pulsar timing consortium" described above) has been organized for the purpose of carrying out follow-up radio observations of any newly-discovered Fermi gamma-ray pulsars. As described in the introduction, one of the main objectives of the mission is to carry out population studies of radio-loud and radio-quiet pulsars, and the first step in this process is to first discover new radio-quiet (or radio-faint) gamma-ray pulsars.

The term "blind" in these searches refers to the fact that the spin parameters of the potential pulsar (e.g. frequency, $f$, and frequency derivative, $\dot{f}$ ) are unknown 2 . Although the location is also technically unknown, it is taken to be that of an astrophysical source, rather than a random point in the sky. In this respect, a more accurate (though awkward) term might be "blind frequency search". Only a relatively small number of locations in the sky is searched (a few hundred in the first few months). The arrival times of

${ }^{2}$ Fortunately, unlike radio searches, gamma-ray searches need not be concerned with searching over a range of values of dispersion measure (DM). 
photons must be corrected to the Solar System barycenter assuming this location, and for each source, a large parameter space in $f$ and $\dot{f}$ must be covered. Using the Crab to define our limiting parameters, a maximum frequency of $64 \mathrm{~Hz}$ was chosen, and a minimum ratio of $\dot{f} / f=-1.25 \times 10^{-11} \mathrm{~s}^{-1}$ (the parameter space is explored in steps of $\dot{f} / f)$. These parameters cover roughly $95 \%$ of the $\sim 2000$ pulsars contained in the ATNF database. Source locations, in general, are derived from the LAT itself. These are mostly new Fermi-LAT gamma-ray unidentified sources, though many are coincident with old EGRET unidentified sources, only much better localized. However, there is another category of source locations used in the pulsar blind searches which correspond to well-studied astrophysical sources which, with various degrees of confidence, are suspected of being pulsars. These are often referred to as "Geminga candidates", and a list of such sources was compiled before the launch of Fermi. In addition to being available straight away (unlike the Fermi-LAT locations which take time to generate, and rely on the constant accumulation of data to improve such locations), these source locations also have the advantage of being obtained mostly at other wavelengths (e.g. Xrays), with much better angular resolution, potentially leading to more sensitive search for pulsations. At the top of the list of "Geminga candidates" are CTA 1 [12] and 3EG 1835+59 (aka the 'next' Geminga) [13], both of which benefitted from pointed observations during the comissioning phase (leading to three times as many photons accumulated over a similar time period in survey mode). In addition to these two, blind searches have been carried out on a number of other sources of interest, such as compact objects in Pulsar Wind Nebulae (PWNe), or TeV sources (e.g. those discovered by HESS or by the Milagro experiment [14]). In fact, the "Dragonfly" pulsar was independently discovered in a blind search on the location of the TeV source MGRO J2019+37.

\section{The time-differencing technique}

As described in the previous section, the low fluxes typical of gamma-ray sources result in very long, sparse data sets. The application of traditional FFT techniques on such data quickly become unfeasible. First, the frequency resolution from such long viewing periods results in FFTs with billions (or tens of billions) of frequency bins. More importantly, the relatively large frequency derivatives of pulsars means that these FFTs would have to be computed a large number of times in order to span a realistic parameter space, given the small step size in $\dot{f}$ required to keep the signal power within a single FFT bin. To make matters worse, the long viewing periods increase the chances that the pulsar will experience a glitch during this time. A new technique, known as "time-differencing" [15, 16], has been developed, which greatly improves the prospects of carrying out these searches. Based on the premise that a periodic signal present in a time series should also show up when analyzing the differences of these times, the method involves taking time differences only up to a short time window (on the order of days, rather than months or years). In doing this, the required number of FFT bins, $N$, is greatly reduced $\left(N=2 \times f \times T_{w}\right.$, where $f$ represents the maximum frequency searched and $T_{w}$ is the maximum time-difference window). The reduced frequency resolution also results in a larger step size required for $\dot{f}$, greatly reducing the number of $\dot{f}$ trials needed. 

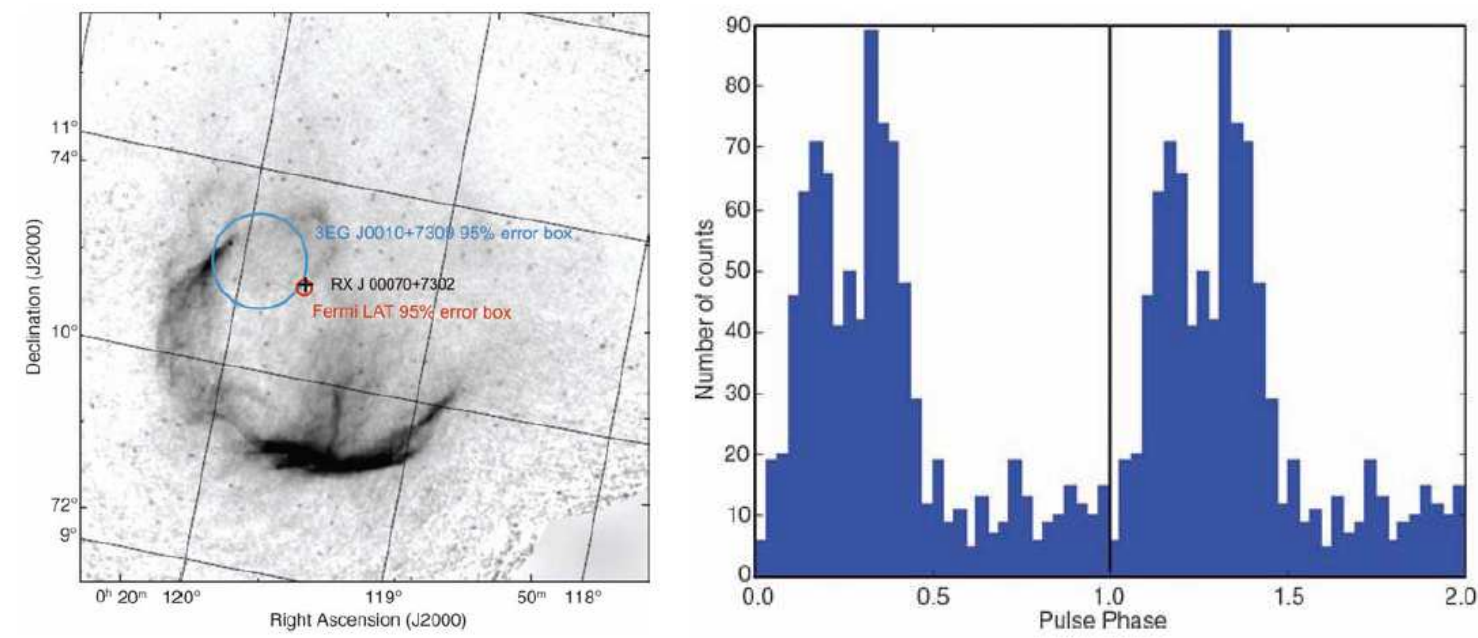

FIGURE 7. Left - Fermi-LAT gamma-ray source location of CTA 1, superimposed on a 1420-MHz radio image of the CTA 1 SNR. The red circle shows the Fermi-LAT 95\% containment radius, while the cross represents the location of the X-ray point source. The large blue circle shows the corresponding EGRET 95\% error circle (taken from [17]). Right - Gamma-ray ( $>100 \mathrm{MeV}$ ) folded light curve of the CTA 1 pulsar shown with two periods of rotation and 32 time bins per period (from [17]).

The overall result is that the computational and memory costs become a fraction of the standard FFT methods, with only a small reduction in sensitivity. This makes it possible to carry out a large number of blind searches over a realistic parameter space on standard desktop computers with only a few GB of memory. That said, the blind search efforts have been significantly enhanced by access to the UCSC Astrophysics Supercomputer Pleiades, with 207 Dell PowerEdge 1950 compute nodes (828 processing cores). Despite the efficiency of the time-differencing technique, it is still desirable to limit the number of source locations in the sky that are searched, given both the finite computational resources available, and also to avoid incurring large penalties in the significance of our detections, due to the increasing number of trials used in finding a particular signal.

\section{A RADIO-QUIET PULSAR IN CTA 1}

The first major Fermi discovery (now published in Science) came with the detection, in a blind search, of pulsations from CTA 1. This young, nearby, shell-type SNR was discovered in radio in the 1960s and X-ray observations show a well-localized central point source, RXJ0007.0+7303, embedded in a pulsar wind nebula (PWN) [12]. High energy $(>100 \mathrm{MeV}$ ) emission was detected by EGRET from 3EG J0010+7309, coincident with this source (see Figure 7), but the low number of photons and poor localization made the search for pulsations very challenging. The new pulsar, with a period of 315.8637050 $\mathrm{ms}$ and a period derivative of $3.615 \times 10^{-13} \mathrm{~s} \mathrm{~s}^{-1}$ is a typical young, energetic pulsar, with a derived characteristic age of $\sim 14,000$ years (consistent with the estimated age of the SNR) and a spin-down power of $4.5 \times 10^{35} \mathrm{erg} \mathrm{s}^{-1}$. For more details, see [17]. 


\section{CONCLUSIONS}

The Fermi Gamma-ray Space Telescope has been performing flawlessly since its launch last June. One of its key scientific missions is to resolve the gamma-ray sky, identifying many (if not all) the gamma-ray sources that its predecessor, EGRET, left unidentified. Many of these EGRET unidentified sources are expected to be pulsars. The hugely improved sensitivity and angular resolution of Fermi, combined with a new powerful time-differencing technique, makes it, for the first time, possible to carry out sensitive searches for gamma-ray pulsations from all these sources. Even before the commissioning phase was complete, Fermi has already yielded important scientific discoveries, including the detection of gamma-ray pulsations from the "Dragonfly" pulsar (PSR J2021+3651), the detection of the first gamma-ray glitch from PSR B1706-44, and the discovery of a new radio-quiet gamma-ray pulsar, in the SNR CTA 1. This last discovery, published in Science, represents a major milestone, as it is the first time that a pulsar has been discovered using gamma-ray observations alone. Based on these early results, Fermi is already fulfilling its pre-launch expectations and is beginning to pave the way for a new era in gamma-ray astrophysics in general, and pulsar astrophysics in particular.

\section{ACKNOWLEDGMENTS}

I want to thank the organizers for putting together a wonderful conference, where the quality of the talks was matched only by that of the food. In particular, I am grateful to Denis Bastieri and Riccardo Rando for all their work and hospitality during the conference, as well as for their efforts (and patience) in the preparation of this volume.

The Fermi LAT Collaboration acknowledges the generous support of a number of agencies and institutes that have supported the Fermi LAT Collaboration. These include the National Aeronautics and Space Administration and the Department of Energy in the United States, the Commissariat à l'Energie Atomique and the Centre National de la Recherche Scientifique / Institut National de Physique Nucléaire et de Physique des Particules in France, the Agenzia Spaziale Italiana, the Istituto Nazionale di Fisica Nucleare (INFN), and the Instituto Nazionale di Astrofisica (INAF) in Italy, the Ministry of Education, Culture, Sports, Science and Technology (MEXT), High Energy Accelerator Research Organization (KEK) and Japan Aerospace Exploration Agency (JAXA) in Japan, and the K. A. Wallenberg Foundation, the Swedish Research Council, and the Swedish National Space Board in Sweden. I am grateful for the support of the American Astronomical Society and the National Science Foundation in the form of an International Travel Grant, which enabled me to attend this conference.

Finally, I would like to dedicate this article to the memory of my brother, Carlos Roberto Saz Parkinson.

\section{REFERENCES}

1. D. J. Thompson, Reports on Progress in Physics 71, 116901-+ (2008), 0811.0738.

2. K. P. Watters, R. W. Romani, P. Weltevrede, and S. Johnston, ArXiv e-prints (2008), 0812.3931. 
3. P. L. Gonthier, R. Van Guilder, and A. K. Harding, ApJ 604, 775-790 (2004), arXiv:astro-ph/0312565

4. W. B. Atwood et al., ApJ (2009).

5. A. A. Abdo et al., ArXiv e-prints (2008), 0812.2960 .

6. M. Ruderman, ApJ 203, 213-222 (1976).

7. A. Krawczyk, A. G. Lyne, J. A. Gil, and B. C. Joshi, MNRAS 340, 1087-1094 (2003), arXiv:astro-ph/0302585

8. S. Johnston, R. N. Manchester, A. G. Lyne, V. M. Kaspi, and N. D’Amico, A\&A 293, 795-802 (1995).

9. D. A. Smith, L. Guillemot, F. Camilo, I. Cognard, D. Dumora, C. Espinoza, P. C. C. Freire, E. V. Gotthelf, A. K. Harding, G. B. Hobbs, S. Johnston, V. M. Kaspi, M. Kramer, M. A. Livingstone, A. G. Lyne, R. N. Manchester, F. E. Marshall, M. A. McLaughlin, A. Noutsos, S. M. Ransom, M. S. E. Roberts, R. W. Romani, B. W. Stappers, G. Theureau, D. J. Thompson, S. E. Thorsett, N. Wang, and P. Weltevrede, $A \& A$ 492, 923-931 (2008), 0810.1637.

10. M. S. E. Roberts, J. W. T. Hessels, S. M. Ransom, V. M. Kaspi, P. C. C. Freire, F. Crawford, and D. R. Lorimer, ApJL 577, L19-L22 (2002), arXiv: astro-ph/0206443.

11. J. P. Halpern, F. Camilo, A. Giuliani, E. V. Gotthelf, M. A. McLaughlin, R. Mukherjee, A. Pellizzoni, S. M. Ransom, M. S. E. Roberts, and M. Tavani, ApJL 688, L33-L36 (2008), 0810.0008 ,

12. J. P. Halpern, E. V. Gotthelf, F. Camilo, D. J. Helfand, and S. M. Ransom, ApJ 612, 398-407 (2004), arXiv:astro-ph/0404312

13. J. P. Halpern, F. Camilo, and E. V. Gotthelf, ApJ 668, 1154-1157 (2007), 0707.1547 .

14. A. A. Abdo, B. Allen, D. Berley, S. Casanova, C. Chen, D. G. Coyne, B. L. Dingus, R. W. Ellsworth, L. Fleysher, R. Fleysher, M. M. Gonzalez, J. A. Goodman, E. Hays, C. M. Hoffman, B. Hopper, P. H. Hüntemeyer, B. E. Kolterman, C. P. Lansdell, J. T. Linnemann, J. E. McEnery, A. I. Mincer, P. Nemethy, D. Noyes, J. M. Ryan, P. M. Saz Parkinson, A. Shoup, G. Sinnis, A. J. Smith, G. W. Sullivan, V. Vasileiou, G. P. Walker, D. A. Williams, X. W. Xu, and G. B. Yodh, ApJL 664, L91-L94 (2007), 0705.0707

15. W. B. Atwood, M. Ziegler, R. P. Johnson, and B. M. Baughman, ApJL 652, L49-L52 (2006).

16. M. Ziegler, B. M. Baughman, R. P. Johnson, and W. B. Atwood, ApJ 680, 620-626 (2008).

17. A. A. Abdo, M. Ackermann, W. B. Atwood, L. Baldini, J. Ballet, G. Barbiellini, M. G. Baring, D. Bastieri, B. M. Baughman, K. Bechtol, R. Bellazzini, B. Berenji, R. D. Blandford, E. D. Bloom, G. Bogaert, E. Bonamente, A. W. Borgland, J. Bregeon, A. Brez, M. Brigida, P. Bruel, T. H. Burnett, G. A. Caliandro, R. A. Cameron, P. A. Caraveo, P. Carlson, J. M. Casandjian, C. Cecchi, E. Charles, A. Chekhtman, C. C. Cheung, J. Chiang, S. Ciprini, R. Claus, J. Cohen-Tanugi, L. R. Cominsky, J. Conrad, S. Cutini, D. S. Davis, C. D. Dermer, A. de Angelis, F. de Palma, S. W. Digel, M. Dormody, E. do Couto e Silva, P. S. Drell, R. Dubois, D. Dumora, Y. Edmonds, C. Farnier, W. B. Focke, Y. Fukazawa, S. Funk, P. Fusco, F. Gargano, D. Gasparrini, N. Gehrels, S. Germani, B. Giebels, N. Giglietto, F. Giordano, T. Glanzman, G. Godfrey, I. A. Grenier, M.-H. Grondin, J. E. Grove, L. Guillemot, S. Guiriec, A. K. Harding, R. C. Hartman, E. Hays, R. E. Hughes, G. Jóhannesson, A. S. Johnson, R. P. Johnson, T. J. Johnson, W. N. Johnson, T. Kamae, Y. Kanai, G. Kanbach, H. Katagiri, N. Kawai, M. Kerr, T. Kishishita, B. Kiziltan, J. Knödlseder, M. L. Kocian, N. Komin, F. Kuehn, M. Kuss, L. Latronico, M. Lemoine-Goumard, F. Longo, V. Lonjou, F. Loparco, B. Lott, M. N. Lovellette, P. Lubrano, A. Makeev, M. Marelli, M. N. Mazziotta, J. E. McEnery, S. McGlynn, C. Meurer, P. F. Michelson, T. Mineo, W. Mitthumsiri, T. Mizuno, A. A. Moiseev, C. Monte, M. E. Monzani, A. Morselli, I. V. Moskalenko, S. Murgia, T. Nakamori, P. L. Nolan, E. Nuss, M. Ohno, T. Ohsugi, A. Okumura, N. Omodei, E. Orlando, J. F. Ormes, M. Ozaki, D. Paneque, J. H. Panetta, D. Parent, V. Pelassa, M. Pepe, M. Pesce-Rollins, G. Piano, L. Pieri, F. Piron, T. A. Porter, S. Rainò, R. Rando, P. S. Ray, M. Razzano, A. Reimer, O. Reimer, T. Reposeur, S. Ritz, L. S. Rochester, A. Y. Rodriguez, R. W. Romani, M. Roth, F. Ryde, H. F.-W. Sadrozinski, D. Sanchez, A. Sander, P. M. Saz Parkinson, T. L. Schalk, A. Sellerholm, C. Sgrò, E. J. Siskind, D. A. Smith, P. D. Smith, G. Spandre, P. Spinelli, J.-L. Starck, M. S. Strickman, D. J. Suson, H. Tajima, H. Takahashi, T. Takahashi, T. Tanaka, J. B. Thayer, J. G. Thayer, D. J. Thompson, S. E. Thorsett, L. Tibaldo, D. F. Torres, G. Tosti, A. Tramacere, T. L. Usher, A. Van Etten, N. Vilchez, V. Vitale, P. Wang, K. Watters, B. L. Winer, K. S. Wood, H. Yasuda, T. Ylinen, and M. Ziegler, Science 322, 1218-(2008). 